\title{
Anatomical templates of lymph node dissection for upper tract urothelial carcinoma: a systematic review of the literature
}

\author{
Riccardo Campi, Andrea Minervini, Andrea Mari, Georgios \\ Hatzichristodoulou, Francesco Sessa, Albero Lapini, Maurizio Sessa, Jurgen \\ Erich Gschwend, Sergio Serni, Marco Roscigno \& Marco Carini
}

To cite this article: Riccardo Campi, Andrea Minervini, Andrea Mari, Georgios

Hatzichristodoulou, Francesco Sessa, Albero Lapini, Maurizio Sessa, Jurgen Erich Gschwend, Sergio Serni, Marco Roscigno \& Marco Carini (2017): Anatomical templates of lymph node dissection for upper tract urothelial carcinoma: a systematic review of the literature, Expert Review of Anticancer Therapy, DOI: 10.1080/14737140.2017.1285232

To link to this article: http://dx.doi.org/10.1080/14737140.2017.1285232

+ View supplementary material $\longleftarrow$

Submit your article to this journal
Accepted author version posted online: 19 Jan 2017.

Published online: 06 Feb 2017.

Q View related articles $\square$

View Crossmark data \lceil 


\title{
Anatomical templates of lymph node dissection for upper tract urothelial carcinoma: a systematic review of the literature
}

\author{
Riccardo Campi $\mathbb{C}^{\mathrm{a}}$, Andrea Minervini ${ }^{\mathrm{a}}$, Andrea Maria, Georgios Hatzichristodoulou ${ }^{\mathrm{b}}$, Francesco Sessa ${ }^{\mathrm{a}}$, Albero Lapini ${ }^{\mathrm{a}}$ \\ Maurizio Sessac, Jurgen Erich Gschwend ${ }^{b}$, Sergio Serni ${ }^{a}$, Marco Roscigno ${ }^{d}$ and Marco Carini ${ }^{a}$

\begin{abstract}
aDepartment of Urology, Careggi Hospital, University of Florence, Florence, Italy; ${ }^{b}$ Department of Urology, Klinik und Poliklinik für Urologie, Technische Universität München (TUM), Klinikum rechts der Isar, Munich, Germany; 'Department of Experimental Medicine, Section of Pharmacology 'L. Donatelli', II University of Naples, Naples, Italy; 'Department of Urology, AO Papa Giovanni XXIII, Bergamo, Italy
\end{abstract}

\section{ABSTRACT}

Introduction: Indications and techniques of lymph node dissection (LND) for upper tract urothelial carcinoma (UTUC) are still controversial.

Areas covered: In this study, a systematic review of the English-language literature was performed up to 1 July 2016 using the Medline, Scopus, Cochrane Library and Web of Sciences databases to provide a detailed overview of the most commonly dissected surgical templates of LND for UTUC according to laterality and location of the tumor. Overall, sixteen studies were analyzed. Based on the shared experiences in the scientific literature, the LND template typically included: for right-sided tumors of the renal pelvis, upper third and middle third of the ureter, the renal hilar, paracaval, precaval and retrocaval nodes, while for left-sided tumors the renal hilar, paraaortic and preaortic nodes. For tumors of the lower ureter, an extended pelvic LND was performed in most cases; however, the paracaval, paraaortic or presacral nodes were dissected in selected series.

Expert commentary: LND is not routinely performed at the time of surgery for UTUC and both indication and extent of LND vary among surgeons and institutions. Future high-quality studies are needed to define the most accurate LND templates and to assess their oncological efficacy and surgical morbidity.
ARTICLE HISTORY

Received 25 October 2016 Accepted 18 January 2017

KEYWORDS

Anatomic landmarks; lymphadenectomy; lymph node dissection; template; transitional cell carcinoma; upper tract urothelial carcinoma; UTUC

\section{Introduction}

Regional lymph nodes (LNs) represent the most common metastatic site of upper tract urothelial carcinoma (UTUC). As such, LN metastases represent a critical prognostic factor [1]. LN dissection (LND) has shown to achieve accurate staging [2-4], increased patient survival [2,5-10], possible treatment of micrometastases [8,11,12] and less in-field recurrence [13]. Nonetheless, indications, techniques and outcomes of LND for UTUC are still debated in the current literature [6-8], and its therapeutic benefit remains controversial [4,7,14-17].

A proper LND is not routinely performed at the time of radical nephroureterectomy (RNU), even at high-volume centers $[4,18]$. Key issues to explain this lack of consensus are inadequate preoperative disease staging, lack of risk-adjusted strategies to select patients eligible for LND, and the inherent variability of lymphatic drainage from UTUC [14]. Moreover, there is lack of standardization in the critical steps of LND and in the reporting of LND results among surgeons/Institutions, which is potentially driven by the lack of specific suggestions by the current Guidelines [19]. On this regard, due to the paucity of available evidence, it is almost unknown whether the lack of standardization involves also the anatomical landmarks of dissection $[20,21]$.

Therefore, the aim of this review is to provide a detailed summary of the available studies in literature that provided specific information on the anatomical landmarks and surgical templates of LND for UTUC, according to laterality and location of the primary tumor.

\section{Materials and methods}

\subsection{Search strategy}

A systematic review of the English-language literature was performed according to the Preferred Reporting Items for Systematic Reviews and Meta-Analyses (PRISMA) criteria [22]. The Medline, Scopus, Cochrane Library and Web of Sciences databases were screened without time limit up to 1 July 2016 using the keywords 'lymph node dissection' or 'lymphadenectomy' or 'lymph nodes' or 'lymphatic drainage' AND 'template' or 'landmarks' or 'mapping' and 'upper tract urothelial carcinoma' or 'UTUC' (Search Query: ((lymph node dissection) or (lymphadenect*) or (lymph node*) or (lymphatic drainage) or landmarks or template or mapping)) and ((upper tract) and ((urothelial carcinoma) or (UTUC) or (transitional cell carcinoma))). In addition, the reference list of each selected original articles and previous review articles about this topic were used to screen for further eligible articles. Two reviewers (R.C. and A.M.) carried out this process independently. The list of articles that were judged highly 
relevant by these authors was reviewed by all co-authors until a final consensus based on a majoritarian system was reached for the articles to be included.

\subsection{Study selection}

All selected studies were screened to retrieve information on the surgical template of LND, the laterality and the location of the primary tumor as well as the number of LNs removed and of LN metastases (LNM) in each site of the template. All the studies that described the execution of surgical treatment of UTUC without LND dissection were excluded. Moreover, those studies with the lack of information on the anatomical boundaries of LND were also excluded.

\subsection{Data extraction}

A data extraction form was developed a priori in order to collect relevant information on study design and level of evidence, number of the patients included, the selection criteria for LND, standardized definition of LND templates, and LND metrics (number of LNs removed). Moreover, relevant information on the number of LNMs, number of LNs dissected in each anatomical site of the template), UTUC characteristics (laterality and location in the upper urinary tract), and surgical approach were collected (Table 1).

A specific electronic extraction sheet was used to collect detailed information regarding the anatomical landmarks of LND according to laterality (right vs. left-sided) and location (renal pelvis (RP), and upper, middle, and lower third of the ureter) of the index UTUC (Table 2). In this regard, upper, middle, and lower ureter were defined as 'superior to the inferior mesenteric artery (IMA)', 'from the level of IMA to the crossing of common iliac artery' and 'below the crossing of common iliac artery' respectively [14].

\section{Results}

In total, 702 articles were preliminarily identified. After exclusion of duplicates generated by the re-execution of the research query in multiple databases $(n=365)$ and the exclusion of papers without specific information on LND templates and/or outcomes $(n=270), 76$ papers were selected. A second screening phase was performed for these studies, which identified overlapping surgical series $(n=5)$, previous reviews of this topic $(n=13)$ and studies that did not fulfill the predefined quality criteria $(n=33)$. In total, 16 studies recruiting 1705 patients fulfill all the inclusion criteria and were selected for this review (Figure 1).

\subsection{Type of studies and quality of reporting LND results}

The information provided in each study, including quality assessment measures, are listed in Table 1. Out of 16 studies that met the inclusion criteria for this review, 14 were retrospective cohort studies, of which nine single center and five multicenter, and two were prospective cohort studies (1 single- and 1 multicenter). Only two studies were specifically designed to determine the primary patterns of lymphatic spread from UTUC and the incidence of LNMs in each anatomical site of the template (mapping studies) [23-25]. Sample size, tumor location, and stage were inhomogeneous throughout the studies. All studies described an open surgical approach for both RNU and LND. A minimally invasive approach was used in some series, using either a laparoscopic $(n=7)$ and/or robotic approach $(n=2)$.

In almost all studies, the author did not define a priori specific selection criteria for LND, being the choice to perform LND left to the surgeons' judgment and/or based on preoperative/intraoperative suspicion of LNMs. Moreover, in most studies the extent of LND was not standardized. In particular, some studies described a standard template of dissection according to either laterality [26$28]$ or location $[7,8,23,29]$ of the primary tumor. Only a few series used a standardized template according to both, tumor laterality and location $[10,12,24,25]$. The overall number of $L N s$ removed in each study is shown in Supplementary Figure 1; however, due to the lack of standardized templates of LND, the number of LNs dissected and LNMs in each anatomical site were rarely reported in the published series.

\subsection{Templates of LND for UTUC of the RP and upper/ middle third of the ureter}

For tumors of the right renal pelvis (RP), upper ureter (UU), and middle ureter (MU), the LND template included in the majority of the studies the renal hilar, precaval, and paracaval nodes $[3,7,8,10,12,23-25,27,29-31]$, extending the dissection to the posterior aspect of the inferior vena cava by transection of the lumbar veins, similar to retroperitoneal $\mathrm{LN}$ dissection for testicular tumors [27], in order to dissect the retrocaval nodes (Figure 2). For left-sided tumors, the LND template included in most cases the renal hilar, pre- and paraaortic nodes $[3,7,8,12,23-25,27,29-$ 31] (Figure 3, Table 2). Discrepancies were found among the selected series on some specific anatomical sites included in the template and on the lower boundary of LND, such as the dissection of interaortocaval nodes, lower boundary of dissection (IMA vs. AB) and the dissection of the pelvic iliac nodes (especially for MU-UTUCs) (Figure 2). For RP-UTUC, the removal of interaortocaval nodes was considered not commonly accepted among the studies, especially for left-sided tumors [10,12,25,27,28,31]. Matin et al. showed the apparent secondary involvement of interaortocaval nodes suggesting the possible role of intraoperative frozen section analysis to omit dissection of these nodes in the case of negative paracaval/retrocaval (for the right side) or paraaortic (left side) areas [25]. In contrast, a significant proportion of LNMs was found in the interaortocaval region in case of MU-UTUCs [25]. Moreover, the same authors outlined the constant presence of out-of-field positive LNs (such as suprahilar, common iliac, aortic bifurcation, and others (Table 2) [25] in case of primary LNMs in the hilar or paravascular areas.

For RP and UU-UTUC, most studies proposed the level of IMA as the lower boundary of dissection [7-9,29,30]. However, some authors extended the dissection along the great vessels until the level of $A B[12,23,24]$ or of the vena cava bifurcation (for rightsided tumors only, [25]). For MU-UTUCs, a high variability was found regarding the lower limit of LND proposed by different authors. Most studies extended the dissection beyond the $A B$ to include also the common $[3,7-9,23,25,29,30,32]$ and external [25], or internal [32] iliac nodes. In one study, the dissection template included only the ipsilateral pelvic LNs [27]. 


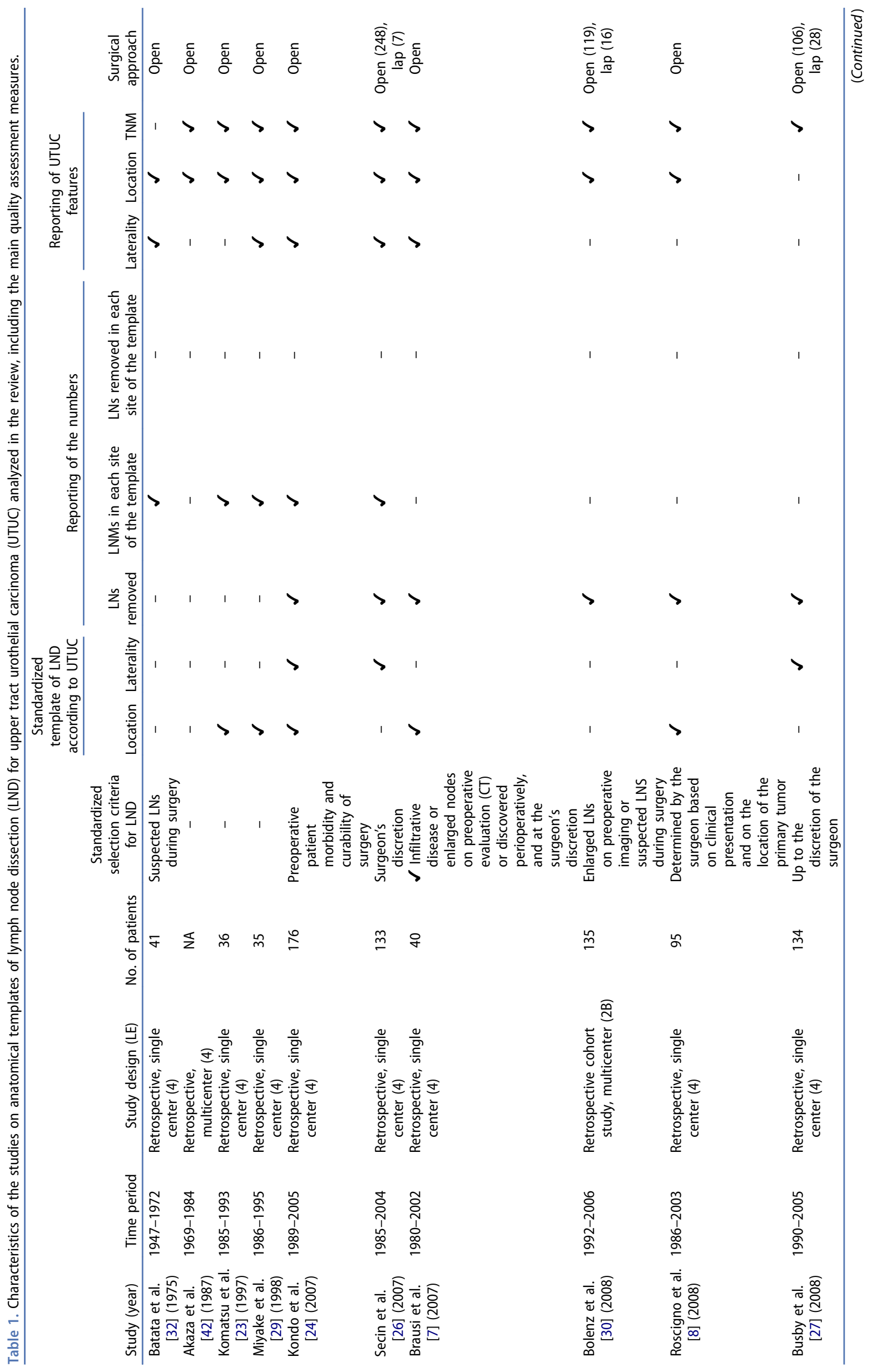









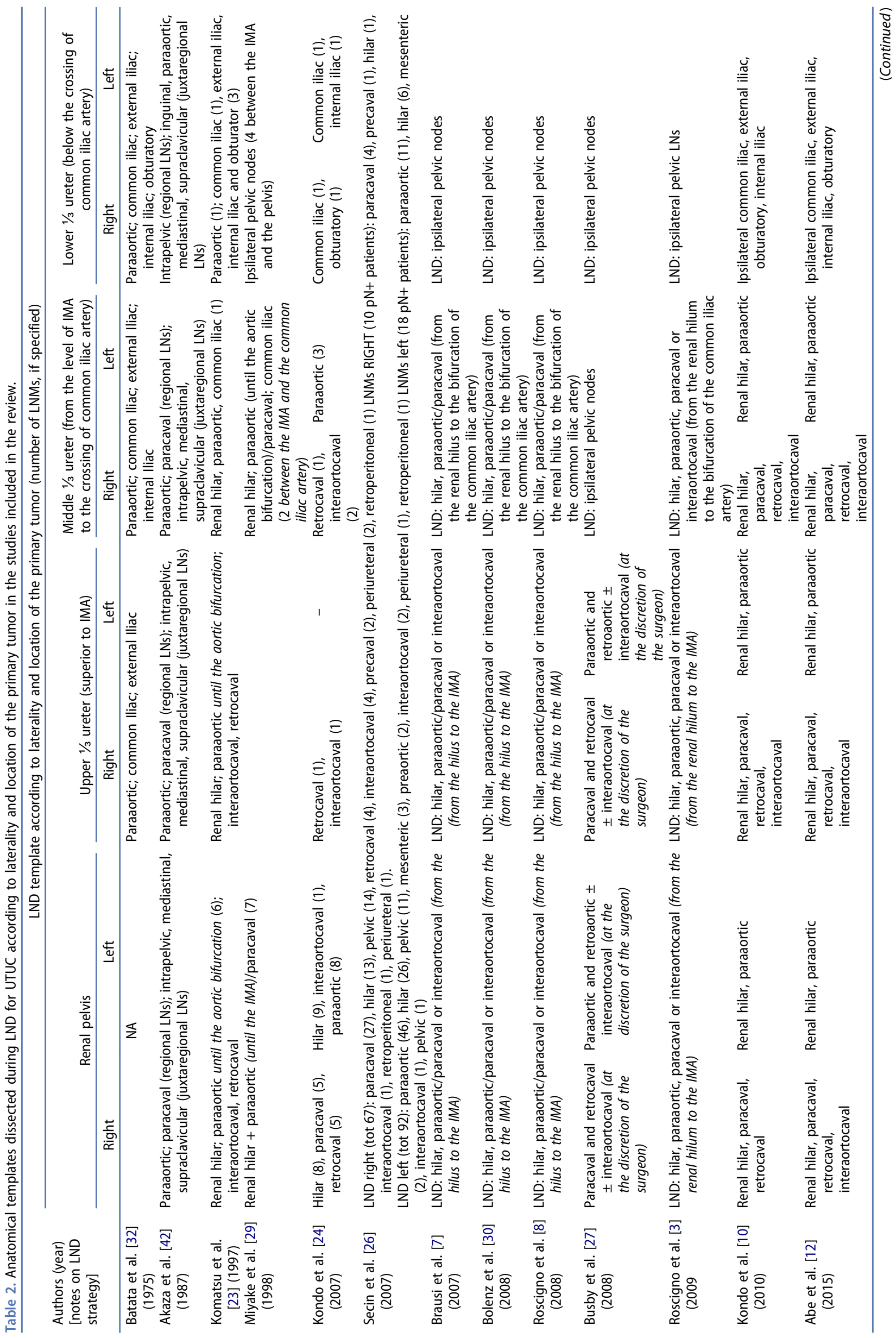




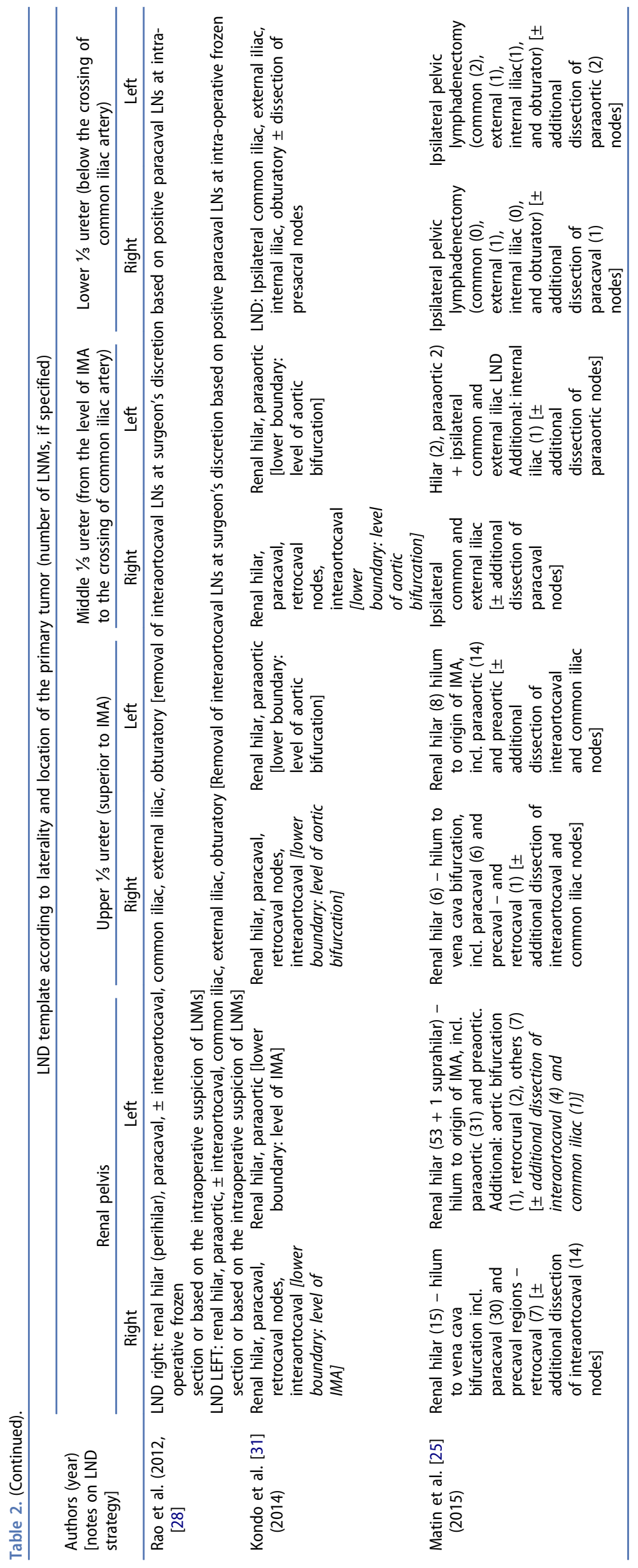




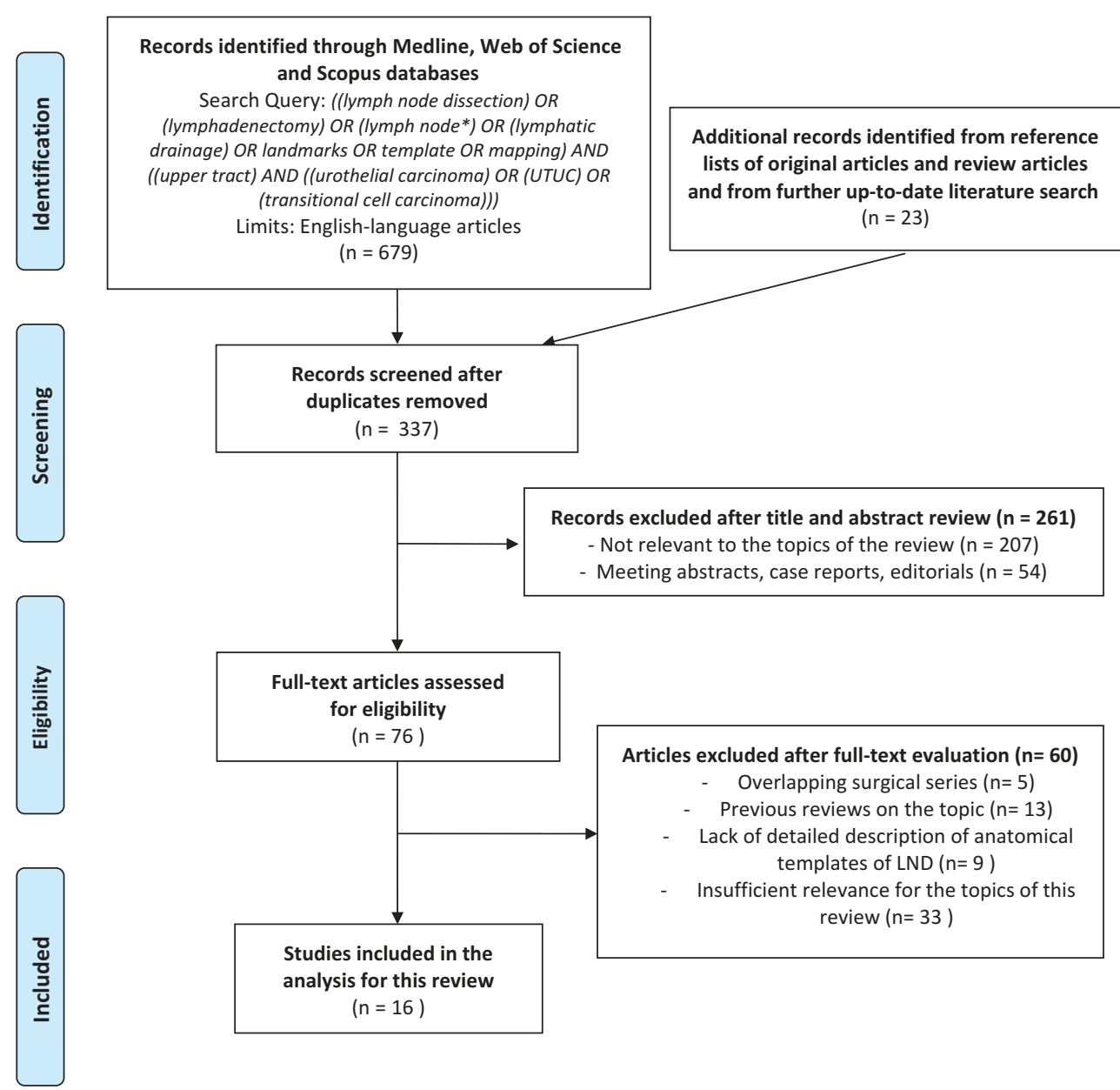

Figure 1. Literature search and study selection process according to the Preferred Reporting Items for Systematic Reviews and Meta-Analyses (PRISMA) statement criteria.

\subsection{LND template for UTUC of the lower third of ureter}

In most studies, the LND template for tumors of the lower third of ureter included only the ipsilateral pelvic LNs (common, external, internal iliac and obturator nodes) (Figure 3, Table 2). Of note, Matin et al. reported the upward migration of metastases to the paracaval and paraaortic regions from mid and distal ureteral tumors [25]. As such, some studies included the paracaval (for right-sided tumors) [25], paraaortic (for left-sided tumors) $[23,25,32]$, or presacral nodes [10] in the template.

\section{Discussion}

\subsection{Rationale for LND at the time of RNU for UTUC}

Urothelial carcinoma of the bladder and of upper urinary tract represent two distinct diseases in terms of anatomical, biological and molecular features [33]. While the prognostic role of LND at radical cystectomy is significantly supported by the evidence and the anatomical templates have been extensively evaluated [34,35], on the contrary LND at the time of RNU has not gained the same oncological role.

In this regard, many studies evaluating the impact of LND for bladder cancer outlined that extended pelvic lymphadenectomy can be curative in patients with metastasis or micro-metastasis to a few nodes and that there is a need for standardization of
LND templates during $\mathrm{RC}$ in order to improve surgical quality and $B C$ patient survival [36,37]. The same conclusions were reached by Bruins et al that outlined the oncologic advantages of performing an extended versus limited LND [38]. Most importantly, a recent paper elegantly showed that, as long as the surgeon adheres to a standardized LND template, the LN count does not affect long-term survival after RC [39]. Therefore, as claimed by many authors $[31,39,40]$, LN count might be inaccurate to define a proper LND, being a better surrogate of the quality of dissection, rather than its extent [41]. As such, only a meticulous dissection within well-defined anatomical boundaries can ensure completeness of LND to optimize the oncological outcomes.

All these findings from the literature on $B C$ provide the rationale to evaluate whether the anatomical extent of LND does provide an oncological benefit for UTUC.

However, due to the variable lymphatic drainage from UTUC and the lack of well established guidelines recommendations [14], it is still debated within the urological community whether LND may provide an oncological benefit compared to RNU alone [19] and, although a growing body of evidence has evaluated the staging and therapeutic benefit of LND for UTUC, there is currently lack of knowledge on its specific anatomical boundaries and selection criteria. 


\section{Surgical anatomy of lymph node dissection for upper tract urothelial carcinoma}

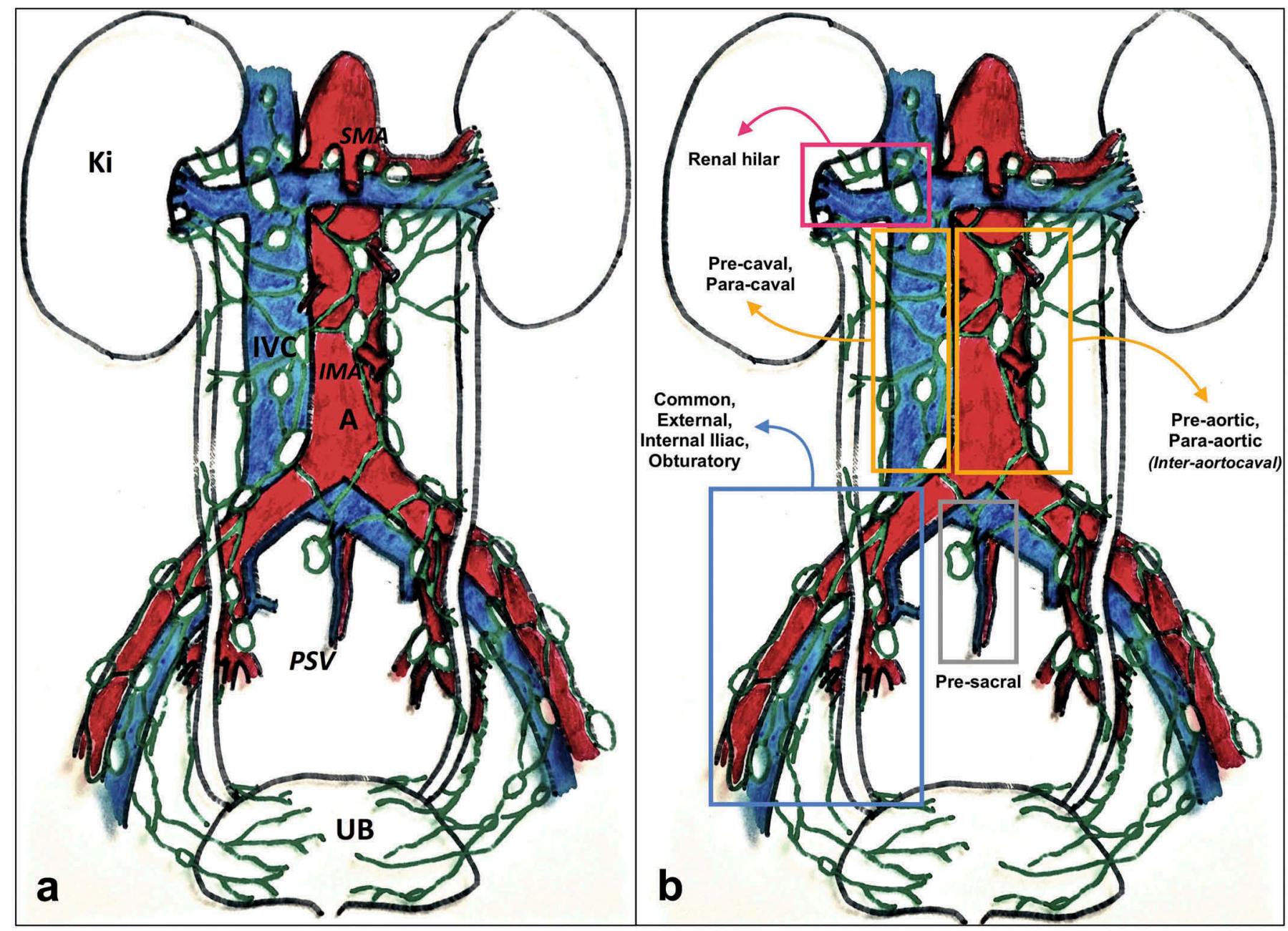

Figure 2. Schematic view of critical anatomical landmarks (left side) and templates (right side) of lymph node dissection for upper tract urothelial carcinoma. A: aorta; IVC: inferior vena cava; GV: gonadal vein; GA: gonadal artery; AB: aortic bifurcation; VCB: vena cava bifurcation; CIA: common iliac artery; EIA: external iliac artery; IIA: internal iliac artery; CIAB: common iliac artery bifurcation; RH: renal hilum; SMI: superior mesenteric artery; IMA: inferior mesenteric artery; Ki: kidney; Ad: adrenal; UB: urinary bladder.

Overall, according to the available evidence, it is still untimely to address whether performing LND for UTUC does provide a clinically significant benefit for ultimate oncologic outcomes of UTUC patients. As such, in the attempt to define the background for future high-quality prospective studies addressing the potential role of lymphadenectomy for UTUC outcomes, we provided a detailed overview of the currently available evidence on anatomical landmarks and surgical templates of LND for UTUC.

\subsection{Selection criteria for LND}

To date, the current Guidelines provided by European Association of Urology (EAU) recommend LND for all invasive UTUCs, without specifying both selection criteria and extent of dissection [14]. In this regard, defining specific selection criteria for extended LND is a primary clinical need as the careful balance between oncological benefits and potential surgical harms of LND is crucial for patient counseling.
In our analysis most studies did not use or report information on the clinical criteria that lead the surgeon to perform LND. In particular, in the studies by Komatsu [23] and Batata [32], despite the authors aimed to assess the survival benefit of LND, no information were reported on the selection criteria for LND. Similarly, Akaza et al and Busby et al. [27,42] did not report any information on the clinical characteristics of UTUC that lead the surgeon to perform LND, although they mentioned whether LND was performed. Contrarily, in the prospective study by Rao et al. [28] that aimed to assess the feasibility of a new technique of retroperitoneal LND for UTUC, it was not reported in the manuscript the potentials reasons for not performing LND. Finally, in the retrospective mapping study conducted by Matin et al., to investigate the patterns of lymphatic spread from UTUC, the authors clearly defined the absence of any information about LND selection criteria as a critical limitation of the study [25].

More importantly, a great inhomogeneity was found also among the eight studies that proposed standardized selection criteria for LND. In one study, the eligibility for LND was decided 


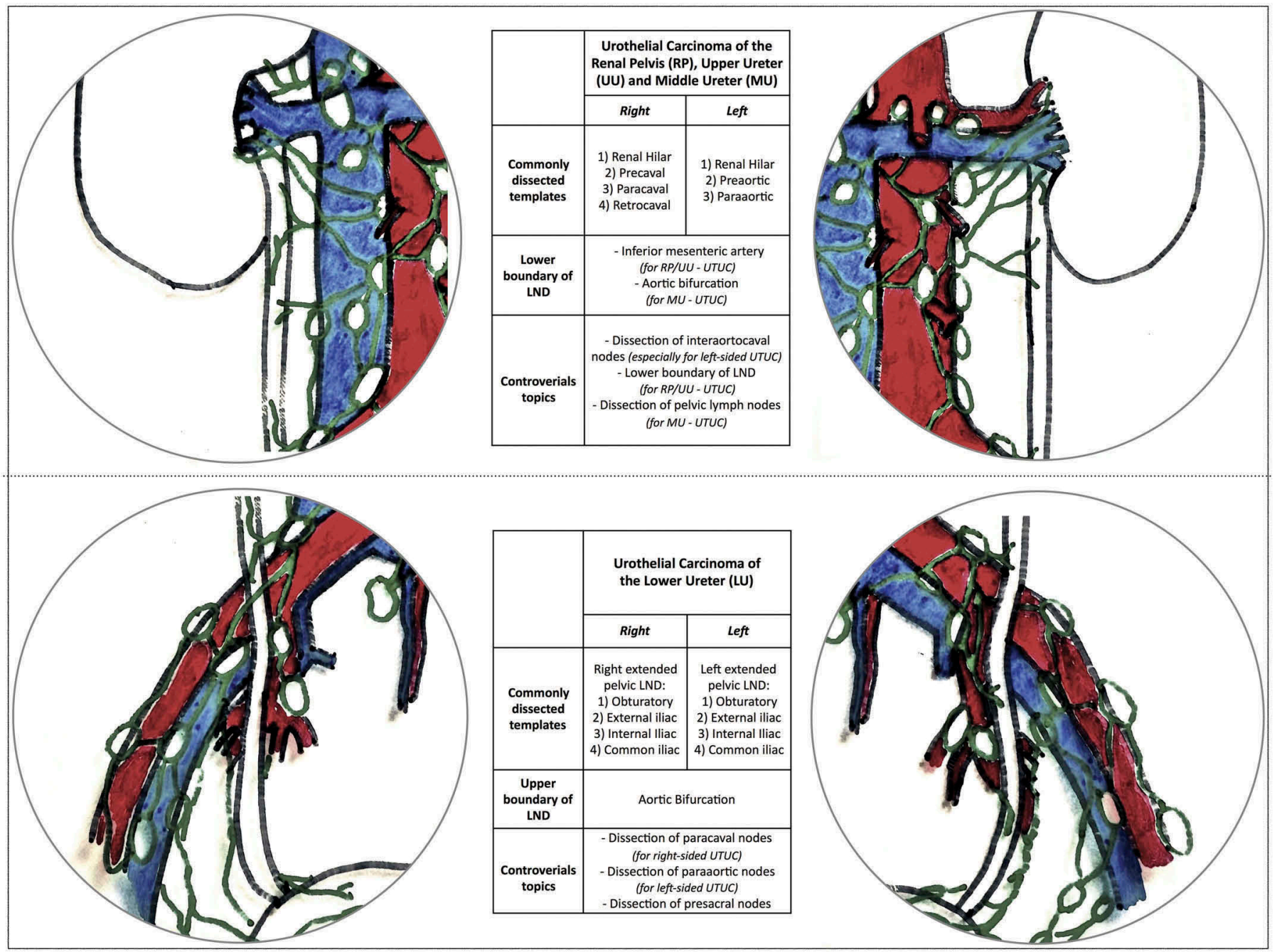

Figure 3. Schematic view of the most commonly dissected templates of lymph node dissection for upper tract urothelial carcinoma of the renal pelvis, upper third, middle third and lower third of the ureter according to laterality of the tumour based on the studies included in our review. The ongoing controversies regarding the anatomical boundaries of dissection are also outlined.

by the treating surgeon during RNU in the absence of any preoperative clinical evaluation [26]. In other studies, the eligibility for LND was based on both the pre-operative (i.e. enlarged LN at the MRI) and intra-operative suspicion of LN metastasis during the surgical procedure (i.e. presence of infiltrative disease or enlarged nodes) and the presence of severe patient comorbidities $[3,7,8,10,11,24,30]$. Finally, in the study conducted by Abe et al., the eligibility for LND was determined by the absence at the preoperative time of apparent $\mathrm{LN}$ swelling, severe hydronephrosis, or perirenal/periureteral invasion [11]. It is important to highlight that in the high volume centers performing routinely LND for UTUC, there was a trend toward a more standardized definition of the selection criteria for LND overtime $[10,24,31]$.

\subsection{Surgical boundaries of LND according to laterality and location}

Our review has shown that the anatomical templates of dissection for UTUC of the renal pelvis (RP), upper ureter (UU) and middle ureter (MU) included in most cases the nodes along the great vessel from the renal hilum to the aortic bifurcation, while for UTUC of the lower ureter an extended pelvic (Figure 3). However, these findings have been obtained by a qualitative analysis of the description of the LND template provided by each study included in our review. To this regard, the lack of a standardized template of LND according to laterality and/or location (or both) of the primary UTUC in most studies $[3,7,8,23,26-30,32,42]$ may hinder the comparison of the available surgical series and the interpretation of their results. Moreover, only in few series the specific number of LNs removed an LNM in each site of the template were accurately reported [10,24-26]. This lack of uniform reporting, together with the lack of standardized anatomical boundaries of LND, may lead to a potentially inaccurate estimation of the specific sites of lymphatic drainage for each anatomical location of UTUC. With regard the surgical approach for RNU and LND, it must be noted that our review was focused on the anatomical templates of lymphadenectomy independently from the surgical approach used. For that reason, our analysis does not provide a truthful overview of the surgical approaches used to perform LND. It was not possible to discuss the feasibility of minimal-invasive approaches for LND in terms of complications 
rate and completeness of dissection since it was not the primary aim of this review. Moreover, it was not possible to assess the complications rate of each anatomical template of LND as the reporting of surgical morbidity of LND was either lacking or unstandardized in most studies included in the review. However, we believe that the relationships between surgical approach (open, laparoscopic, robotic), anatomical template of LND and extent of surgical morbidity is of utmost importance and should be accurately addressed in future prospective studies using a standardized template of dissection.

\subsection{Limitations at a study- and review-level}

This is the first systematic review of the literature specifically designed to provide detailed data on the most commonly dissected surgical templates of LND for UTUC according to both laterality and location of the tumour with the aim to define the current state of the art and the most relevant research needs and future perspectives. However, it does have limitations at both a study- and review-level.

First, many studies analyzed by our review showed several limitations and great inhomogeneity with regard to study design, quality of reporting and standardization of LND templates.

The exclusion of studies addressing the role of LND for UTUC that did not report a detailed description of the anatomical templates of LND may have resulted in selection bias, potentially reducing the generalization of our conclusions. Secondly, even among the finally included in the review, the lack of uniformity in reporting LND templates and the specific site of LNMs may have hindered the understanding of patterns of lymphatic drainage from UTUC (in most studies it was reported only the specific sites of LND and not whether LNMs were present in those areas).

Regarding the limitations of the study design, only two studies included in our analysis were prospective and sample size, tumor location and tumor stage were inhomogeneous throughout the studies. These limitations may prevent an accurate interpretation on available evidence from both a methodological and clinical point of view.

As previously discussed, in almost all studies there were no standardized criteria for LND, being the choice of performing LND left at the surgeons' judgment. Moreover, the extent of LND was not standardized in most series and the exact number of LNs dissected and LNMs in each anatomical site of the template was not reported. Finally, most studies did not specify in a standardized way the exact tumor location and laterality in relation to the LND templates.

\subsection{Future perspectives and research need}

Prospective high-quality studies are required to provide insights on the therapeutic efficacy of LND for UTUC. Thus, standardization in both LND templates and reporting of surgical results is warranted. As such, we believe a more thoughtful reporting of the templates of dissection used and the extension of LND performed will be key to share valuable information between surgeons/Institutions. It should be mentioned that the surgical approach (open, laparoscopic, robot-assisted) should not matter as long as oncological principles and dissection templates are being respected. The overall number of LNs removed, the specific number of LNs dissected and of LNMs in each anatomical site of the template should be reported. The anatomical template should guide the dissection, while the number of LNs removed might provide a summary measure of the dissection quality [6]. Moreover, accurate reporting of tumor location, laterality and staging (both, clinical and pathological TNM classification) should be provided in future series. Finally, a detailed description of the complication rates associated with each dissection template and type of surgical approach using standardized instruments should be added. This aspect will be key to evaluate indications, oncological benefits and potential comorbidities of each dissection template in a meaningful way.

\section{Expert commentary}

The aim of our review was to collect all the available evidence on the anatomical landmarks and the surgical templates of LND for UTUC according to laterality and location of the tumor as well as the selection criteria for LND.

Based on the shared experiences in the scientific literature, the LND template for UTUC of the upper/middle ureter and renal pelvis typically included the renal hilar, paracaval, precaval, retrocaval nodes and the renal hilar, paraaortic and preaortic nodes for right-sided and left-sided tumors, respectively. For tumors of the lower ureter, an extended pelvic LND involving the common, external, internal iliac and obturatory nodes was performed in most series.

However, LND is not routinely performed at the time of surgery, and in most series both, indication and extent of LND vary among surgeons and institutions.

Future high-quality studies are needed to define the most accurate LND templates and to assess their oncological efficacy and surgical morbidity.

\section{Five-year view}

The current role of lymph node dissection (LND) for ultimate oncologic outcomes of patients with UTUC is still controversial. Consequently, there are currently no objective selection criteria for LND and both surgical techniques and anatomical templates are not standardized among surgeons and Institutions worldwide.

In this complex clinical scenario, future clinical research will have to address two distinct unmet clinical needs. Prospective, well-designed clinical trials will be indeed key to: 1) fill the current gaps of knowledge on the anatomical sites of lymphatic spread from UTUC and 2) understand whether LND does provide a (cancer-specific) survival advantage in patients with UTUC and, if so, define the proper extent of dissection for each tumor location within the upper urinary tract in order to balance oncologic efficacy and surgical morbidity. To reach these goals, the study design will be of paramount importance. Indeed, such trials should (a) use standardized surgical templates with clearly defined anatomical boundaries to have the possibility to perform mapping studies; (b) be able to compare oncologic efficacy and surgical morbidity of different surgical 
approaches (open vs. laparoscopic vs. robotic) by using the same templates among different surgeons/institutions; (c) take into account the increasing role of kidney-sparing surgery versus radical nephroureterectomy for the treatment of UTUC of the lower urinary tract. In this scenario, the real need of randomized controlled trials is still debated, as elegantly expressed by Briganti et al. for LND at the time of radical prostatectomy for prostate cancer [43].

In conclusion, individualized tailoring of both indications and extent of LND will be a key step forward in the treatment of high-risk UTUC in order to maximize the oncological efficacy of surgery while reducing its potential morbidity. While waiting the results of future prospective trials, standardization of LND templates according to laterality and location of the tumor and accurate reporting of LND results within the published series is needed to improve the quality of clinical research in this field.

\section{Key issues}

- According to the current evidence, lymph node dissection (LND) has shown to improve staging, patient survival and treatment of micro-metastases in patients with upper tract urothelial carcinoma (UTUC). However, indications and techniques of LND for UTUC are still debated and its therapeutic benefit remains controversial.

- There is lack of standardization in the technique of LND among surgeons and Institutions worldwide, which is potentially driven by the lack of specific Recommendations by the current international Guidelines.

- This is the first systematic review of the literature specifically designed to provide detailed data on the most commonly dissected surgical templates of LND for UTUC according to both laterality and location of the tumour.

- Overall, 16 studies were selected for the analysis. An open surgical approach for both radical nephroureterectomy and LND was used in most studies, while a minimally invasive approach (laparoscopic or robotic) only in selected series.

- In almost all studies, the choice to perform LND was left to the surgeons' judgment and/or was based on preoperative/intraoperative suspicion of lymph node metastases. Moreover, in most studies the extent of LND was not standardized.

- For right-sided tumors of the renal pelvis, upper third and middle third of the ureter, the LND template included in most cases the renal hilar, paracaval, precaval and retrocaval nodes, while for left-sided tumors the renal hilar, paraaortic and preaortic nodes.

- For tumors of the lower ureter, an ipsilateral extended pelvic LND including the common, external, internal iliac and obturatory nodes was performed in most series. However, the paracaval (for right-sided tumors), paraaortic (for left-sided tumors) or the presacral nodes were also dissected in selected studies.

- The lack of a standardized template of LND according to laterality and/or location of UTUC may hinder a proper interpretation of the oncologic results of the available surgical series and a meaningful comparison of different LND techniques, leading to potentially inaccurate estimations of the specific anatomical sites of lymphatic drainage for each anatomical location of UTUC.
- Prospective high-quality studies are required to provide insights on the therapeutic efficacy of LND for UTUC. Thus, standardization of LND templates and thoughtful reporting of LND results will be key in the design of future clinical trials to define the proper templates for each tumor location and to assess their oncological efficacy and surgical morbidity.

\section{Funding}

This article was not funded.

\section{Declaration of interest}

The authors have no relevant affiliations or financial involvement with any organization or entity with a financial interest in or financial conflict with the subject matter or materials discussed in the manuscript. This includes employment, consultancies, honoraria, stock ownership or options, expert testimony, grants or patents received or pending, or royalties.

\section{ORCID}

Riccardo Campi (D) http://orcid.org/0000-0001-5237-0888

\section{References}

Papers of special note have been highlighted as either of interest $(\cdot)$ or of considerable interest $(\cdot \bullet)$ to readers.

1. Lughezzani G, Burger M, Margulis V, et al. Prognostic factors in upper urinary tract urothelial carcinomas: a comprehensive review of the current literature. Eur Urol. 2012;62(1):100-114.

2. Roscigno $M$, Brausi $M$, Heidenreich $A$, et al. Lymphadenectomy at the time of nephroureterectomy for upper tract urothelial cancer. Eur Urol. 2011;60(4):776-783.

3. Roscigno M, Shariat SF, Margulis V, et al. Impact of lymph node dissection on cancer specific survival in patients with upper tract urothelial carcinoma treated with radical nephroureterectomy. J Urol. 2009;181(6):2482-2489.

4. Lughezzani $G$, Jeldres $C$, Isbarn $H$, et al. A critical appraisal of the value of lymph node dissection at nephroureterectomy for upper tract urothelial carcinoma. Urology. 2010;75(1):118-124.

5. Kondo T, Tanabe K. Role of lymphadenectomy in the management of urothelial carcinoma of the bladder and the upper urinary tract. Int J Urol [Internet]. 2012; 19(8):710-721. Available from: http:// www.ncbi.nlm.nih.gov/pubmed/22515472

6. Kondo T, Nakazawa $\mathrm{H}$, Ito $\mathrm{F}$, et al., Impact of the extent of regional lymphadenectomy on the survival of patients with urothelial carcinoma of the upper urinary tract. J. Urol. 2007;178(4Pt 1):1212-1217; discussion 1217

7. Brausi MA, Gavioli M, De Luca G, et al. Retroperitoneal lymph node dissection (RPLD) in conjunction with nephroureterectomy in the treatment of infiltrative transitional cell carcinoma (TCC) of the upper urinary tract: impact on survival. Eur Urol. 2007;52(5):14141420.

8. Roscigno M, Cozzarini C, Bertini R, et al. Prognostic value of lymph node dissection in patients with muscle-invasive transitional cell carcinoma of the upper urinary tract. Eur Urol. 2008;53(4):794-802.

9. Roscigno M, Shariat SF, Margulis V, et al. The extent of lymphadenectomy seems to be associated with better survival in patients with nonmetastatic upper-tract urothelial carcinoma: how many lymph nodes should be removed? Eur Urol. 2009;56(3):512-519.

10. Kondo $T$, Hashimoto $Y$, Kobayashi $H$, et al. Template-based lymphadenectomy in urothelial carcinoma of the upper urinary tract: impact on patient survival. Int J Urol [Internet]. 2010;17:848-854. Available from: http://www.ncbi.nlm.nih.gov/pubmed/20812922 
11. Abe T, Shinohara N, Muranaka M, et al. Role of lymph node dissection in the treatment of urothelial carcinoma of the upper urinary tract: multi-institutional relapse analysis and immunohistochemical re-evaluation of negative lymph nodes. Eur J Surg Oncol. 2010;36 (11):1085-1091.

12. Abe T, Takada N, Matsumoto R, et al. Outcome of regional lymphadenectomy in accordance with primary tumor location on laparoscopic nephroureterectomy for urothelial carcinoma of the upper urinary tract: a prospective study. J Endourol [Internet]. 2015;29:304-309. Available from: http://www.ncbi.nlm.nih.gov/pubmed/25255401

13. Kondo T, Hara I, Takagi T, et al. Possible role of template-based lymphadenectomy in reducing the risk of regional node recurrence after nephroureterectomy in patients with renal pelvic cancer. Jpn J Clin Oncol [Internet]. 2014;44:1233-1238. Available from: http:// www.ncbi.nlm.nih.gov/pubmed/25271269

14. Roupret $M$, Babjuk $M$, Comperat $E$, et al. European association of urology guidelines on upper urinary tract urothelial cell carcinoma: 2015 update. Eur. Urol. 2015;68(5):868-879

15. Stein JP, Skinner DG. The role of lymphadenectomy in high-grade invasive bladder cancer. Urol Clin North Am. 2005;32(2):187-197.

16. Herr HW, Bochner BH, Dalbagni G, et al. Impact of the number of lymph nodes retrieved on outcome in patients with muscle invasive bladder cancer. J Urol. 2002;167(3):1295-1298.

17. Mason RJ, Kassouf W, Bell DG, et al. The contemporary role of lymph node dissection during nephroureterectomy in the management of upper urinary tract urothelial carcinoma: the Canadian experience. Urology. 2012;79(4):840-845.

18. Russo P. Editorial comment. Urology. 2009;74(5):1075-1076.

19. Mandalapu RS, Remzi M, De Reijke TM, et al. Update of the ICUDSIU consultation on upper tract urothelial carcinoma 2016: treatment of low-risk upper tract urothelial carcinoma. World J. Urol [Internet]. 2016. Available from: http://www.ncbi.nlm.nih.gov/ pubmed/27233780

20. Seisen T, Shariat SF, Cussenot O, et al. Contemporary role of lymph node dissection at the time of radical nephroureterectomy for upper tract urothelial carcinoma. World J. Urol [Internet]. 2016. Available from: http://www.ncbi.nlm.nih.gov/pubmed/26809456

- This review outlines an overview of the current evidence on the staging and therapeutic benefit of lymph node dissection for upper tract urothelial carcinoma.

21. Seisen T, Nison L, Remzi M, et al. Oncologic outcomes of kidney sparing surgery versus radical nephroureterectomy for the elective treatment of clinically organ confined upper tract urothelial carcinoma of the distal ureter. J Urol. 2016;195(5):1354-1361

22. Moher D, Liberati A, Tetzlaff J, et al. PRISMA Group. Preferred reporting items for systematic reviews and meta-analyses: the PRISMA statement. Plos Med [Internet]. 2009;6(7):e1000097. Available from: http://www.ncbi.nlm.nih.gov/pubmed/19621072

23. Komatsu H, Tanabe N, Kubodera $S$, et al. The role of lymphadenectomy in the treatment of transitional cell carcinoma of the upper urinary tract. J Urol. 1997;157(5):1622-1624.

24. Kondo $T$, Nakazawa $H$, Ito $F$, et al., Primary site and incidence of lymph node metastases in urothelial carcinoma of upper urinary tract. Urology. 2007;69(2):265-269.

- These are available mapping studies evaluating the anatomic landing sites of lymph node metastases from upper tract urothelial carcinoma.

25. Matin SF, Sfakianos JP, Espiritu PN, et al., Patterns of lymphatic metastases in upper tract urothelial carcinoma and proposed dissection templates. J Urol. 2015;194(6):1567-1574.

- These are available mapping studies evaluating the anatomic landing sites of lymph node metastases from upper tract urothelial carcinoma.

26. Secin FP, Koppie TM, Salamanca JIM, et al. Evaluation of regional lymph node dissection in patients with upper urinary tract urothelial cancer. Int. J. Urol [Internet]. 2007;14:26-32. Available from: http://www.ncbi.nlm.nih.gov/pubmed/17199856

27. Busby JE, Brown GA, Matin SF. Comparing lymphadenectomy during radical nephroureterectomy: open versus laparoscopic. Urology. 2008;71(3):413-416.
28. Rao SR, Correa JJ, Sexton WJ, et al. Prospective clinical trial of the feasibility and safety of modified retroperitoneal lymph node dissection at time of nephroureterectomy for upper tract urothelial carcinoma. BJU Int [Internet]. 2012;110:E475-80. Available from: http://www.ncbi.nlm.nih.gov/pubmed/22564727

29. Miyake H, Hara I, Gohji K, et al. The significance of lymphadenectomy in transitional cell carcinoma of the upper urinary tract. $\mathrm{Br} J$ Urol [Internet]. 1998; 82(4):494-498. Available from: http://www. ncbi.nlm.nih.gov/pubmed/9806176

30. Bolenz C, Shariat SF, Fernández Ml, et al. Risk stratification of patients with nodal involvement in upper tract urothelial carcinoma: value of lymph-node density. BJU Int [Internet]. 2009;103:302-306. Available from: http://www.ncbi.nlm.nih.gov/ pubmed/18990164

31. Kondo T, Hara I, Takagi T, et al. Template-based lymphadenectomy in urothelial carcinoma of the renal pelvis: a prospective study. Int $J$ Urol [Internet]. 2014;21:453-459. Available from: http://www.ncbi. nlm.nih.gov/pubmed/24754341

32. Batata MA, Whitmore WF, Hilaris BS, et al. Primary carcinoma of the ureter: a prognostic study. Cancer [Internet]. 1975; 35 (6):1626-1632. Available from: http://www.ncbi.nlm.nih.gov/ pubmed/1148996

33. Green DA, Rink $M$, Xylinas $E$, et al. Urothelial carcinoma of the bladder and the upper tract: disparate twins. J Urol. 2013;189 (4):1214-1221.

34. Koppie TM, Vickers AJ, Vora $\mathrm{K}$, et al. Standardization of pelvic lymphadenectomy performed at radical cystectomy: can we establish a minimum number of lymph nodes that should be removed? Cancer [Internet]. 2006; 107(10):2368-2374. Available from: http:// www.ncbi.nlm.nih.gov/pubmed/17041887

35. Kassouf W, Svatek RS, Shariat SF, et al. Critical analysis and validation of lymph node density as prognostic variable in urothelial carcinoma of bladder. Urol. Oncol. 2013;31(4):480-486.

36. Karl A, Carroll PR, Gschwend JE, et al. The impact of lymphadenectomy and lymph node metastasis on the outcomes of radical cystectomy for bladder cancer. Eur Urol. 2009;55(4):826-835.

- This recent paper elegantly showed that, as long as the surgeon adheres to a standardized lymph node dissection template, the lymph node count does not affect long-term survival after radical cystectomy.

37. Tilki D, Brausi $M$, Colombo R, et al. Lymphadenectomy for bladder cancer at the time of radical cystectomy. Eur Urol. 2013;64(2):266276.

38. Bruins HM, Veskimae $E$, Hernandez $V$, et al. The impact of the extent of lymphadenectomy on oncologic outcomes in patients undergoing radical cystectomy for bladder cancer: a systematic review. Eur Urol. 2014;66(3):489-499.

39. Fransen Van De Putte EE, Hermans TJN, Werkhoven EV, et al. Lymph node count at radical cystectomy does not influence long-term survival if surgeons adhere to a standardized template. Urol. Oncol. 2015;33(12):504.e19-24.

- This papers outlines how the anatomical template should guide the dissection, while the number of LNs removed might provide a summary measure of the dissection quality.

40. Kondo T, Takagi T, Tanabe K. Therapeutic role of template-based lymphadenectomy in urothelial carcinoma of the upper urinary tract. World J Clin Oncol [Internet]. 2015; 6(6):237-251. Available from: http://www.ncbi.nlm.nih.gov/pubmed/26677437

41. Pohar KS. The anatomic extent and completeness of pelvic lymphadenectomy is what matters. Curr Opin Urol [Internet]. 2013; 23 (5):444-448. Available from: http://www.ncbi.nlm.nih.gov/pubmed/ 23851384

42. Akaza H, Koiso K, Niijima T. Clinical evaluation of urothelial tumors of the renal pelvis and ureter based on a new classification system. Cancer [Internet]. 1987; 59(7):1369-1375. Available from: http:// www.ncbi.nlm.nih.gov/pubmed/3815309

43. Briganti A, Giannarini G, Karnes RJ, et al. What evidence do we need to support the use of extended pelvic lymph node dissection in prostate cancer? Eur Urol. 2015 Apr;67(4):597598 